\title{
Covid-19: Temas de interés para el cirujano
}

\author{
COVID-19: topics of interest to the surgeon \\ Grupo de Médicos Residentes de Cirugía General - Universidad CES'1, Sebastián Sierra-Sierra² \\ 1 Médicos residentes de Cirugía general Universidad CES: María Adelaida Arbeláez-Salgado, Andrés Cadavid-Congote, Daniela Rosa \\ Flórez-Filomeno, Juan Sebastián Garcés-Otero, Agustín Gómez-Machado, Carolina Guzmán-Arango, Daniella Londoño-Restrepo, \\ Carolina Maya-López, Paula Andrea Mejía-Cardona, María Alejandra Molina-Rodríguez, Juan Santiago Molina-Velásquez, Alejandra \\ Ochoa-Pineda, Laura Paredes-Minotas, Mariana Ramírez-Ceballos, Camilo Restrepo-Gómez, María Alejandra Sanín-Osorio, Javier \\ Andrés Serrano-De Castro, Juan José Turizo-Mejía, Andrés Uribe-Valencia, Andrés Velásquez-Hoyos \\ 2 Médico, Especialista en Cirugía General, Clínica CES. Medellín, Colombia
}

\section{Introducción}

Es claro que se está viviendo un momento único en la historia de la humanidad, por una situación que afecta el quehacer diario de todos los trabajadores de la salud y que crea controversias en cuanto al manejo de ciertas patologías. Para tratar de guiar este quehacer diario y tener claridad sobre varios puntos relacionados al COVID-I9 y la patología quirúrgica, se llevaron cabo una serie de reuniones académicas virtuales en las cuales participaron los residentes de Cirugía General de la Universidad CES, el jefe del Programa de Cirugía General, los grupos de Cirugía General y Cirugía Hepatobiliar de la Clínica CES, además de médicos especialistas en Radiología, Medicina Interna y Cirugía Oncológica. El producto fue esta revisión de la literatura con una serie recomendaciones, en la cual se pretende condensar lo que se viene mencionando como protección para el cirujano y todo el equipo quirúrgico, así como las modificaciones en la forma de trabajo durante esta contingencia, teniendo en mente que la información cambia y se actualiza a diario.

Recientemente se publicó el consenso colombiano de atención, diagnóstico y manejo de la infección por SARS-COV-2/COVID-I9 en establecimientos de atención de la salud ${ }^{\mathrm{I}}$, el cual incluye una serie de recomendaciones basadas en un consenso de expertos e informadas en la evidencia. Dentro de las definiciones operativas de un caso sospechoso de infección por COVID-I9 se recomienda diferenciar entre población sintomática y asintomática.

Palabras clave: COVID-I9; virus del SRAS; coronavirus; infecciones por coronavirus; pandemias; cirugía general; quirófanos.

Key words: COVID-I9; SARS virus; coronavirus; coronavirus infections; pandemics; general surgery; operating rooms.

Fecha de recibido: 05/04/2020 - Fecha de aceptación: 07/04/2020 - Fecha de actualización: 27/04/2020

Correspondencia: Sebastián Sierra, MD. Calle 58 N 50C-2 Prado (Clínica CES), Teléfono: 034-5767273.

Correo electrónico: sebastiancirugia@gmail.com

Citar como: Universidad CES, Sierra-Sierra S. COVID-19: Temas de interés para el cirujano. Rev Colomb Cir. 2020;35:153-61. https://doi.org/10.30944/20117582.612

Este es un artículo de acceso abierto bajo una Licencia Creative Commons - BY-NC-ND https://creativecommons.org/licenses/by-ncnd/4.0/deed.es 
La definición operativa de caso sospechoso de infección por SARS CoV-2 / COVID-I9 para personas sintomáticas menciona: persona con síntomas respiratorios agudos (2 o más de los siguientes: tos, dificultad respiratoria, odinofagia, fatiga/adinamia), con presencia o no de fiebre mayor o igual a $38^{\circ} \mathrm{C}$, asociado a:

- Contacto con persona con sospecha o confirmación de infección por SARS $\mathrm{CoV}-2$, o que resida o haya viajado a un área con presentación de casos en los últimos I4 días

- Imágenes pulmonares en vidrio esmerilado periférico o consolidaciones bilaterales

- Persistencia de sintomatología respiratoria o empeoramiento al día 8 desde de su aparición

En cuanto a sospecha de infección en personas asintomáticas, se define una persona que ha tenido contacto estrecho con un caso confirmado de COVID-I9 en los últimos I4 días. Del caso sospechoso se deriva el caso probable, siendo la persona con un cuadro clínico sospechoso o asintomático, asociado a una prueba rápida positiva o una reacción en cadena de polimerasa con transcriptasa reversa (RT-PCR) no concluyente para la identificación de SARS CoV-2 / COVID-I9.

El caso confirmado, sintomático o asintomático, se define como aquel paciente que tiene resultado positivo en alguna de las pruebas moleculares o genómicas. En la guía se recomienda la realización de reacción en cadena de polimerasa con transcriptasa reversa (RT-PCR) de SARS $\mathrm{CoV}-2$ para personas sintomáticas. Si existe alta sospecha y la primera prueba es negativa, se recomienda según disponibilidad, una segunda prueba a las 72 horas I.

Cabe aclarar que el 3i de marzo del 2020, se emitió un comunicado desde el Ministerio de Salud y Protección Social, en el cual afirma que se debe considerar a todo paciente que ingresa a una institución de salud como caso sospechoso para infección por COVID-I9, dado que Colombia se declaró en estado de mitigación. Esto significa que más de un Io\% de los infectados no tienen un nexo epidemiológico ${ }^{2}$.

\section{Evaluación del paciente sospechoso}

En caso de tener un paciente sospechoso de infección por COVID-I9 a nivel intrahospitalario, la guía de la Asociación Colombiana de Infectología (ACIN) recomienda establecer un aislamiento por gotas ${ }^{1}$. El tiempo propuesto idealmente es entre I4 y 28 días, que es el tiempo en que se ha visto de máxima diseminación viral, a menos que se tenga disponible seguimiento con RT-PCR, pues en el momento que se tenga una PCR negativa se considera que el paciente es apto para salir del aislamiento.

Los elementos de protección personal (EPP) incluidos en este tipo de aislamiento son mascarilla quirúrgica convencional, bata antifluido, guantes, protector ocular y gorro (opcional). En caso de practicar procedimientos que generen aerosoles (PGA), como intubación orotraqueal, aspiración de secreciones, fibronasolaringoscopia, fibrobroncoscopia, o endoscopia digestiva, se debe implementar la mascarilla N95.

Existe aún debate sobre si estos pacientes deben tener aislamiento por aerosoles (microorganismos $<5$ micras) o por gotas (> 5 micras). La guía de la ACIN se basa en una revisión sistemática que se publicó en febrero de 2020 en Sichuan, China. Se incluyeron 6 ensayos clínicos aleatorizados, con un total de 9I7I pacientes con influenza confirmada por pruebas de laboratorio y se concluyó que no hay diferencia entre utilizar la mascarilla quirúrgica convencional versus la N95 para prevenir la infección con influenza a nivel intrahospitalario. Estos resultados se extrapolaron al COVID-I9, razón por la cual se opta por el aislamiento por gotas (con mascarilla quirúrgica convencional), a menos que se vaya a practicar un PGA 3 .

La Asociación Española de Cirujanos (AEC), publicó una serie de recomendaciones para examinar al paciente con sospecha de infección por COVID-I9 ${ }^{4}$, que incluyen:

- Dejar todas las pertenencias en zona segura, recogerse el pelo, introducirse la parte superior del uniforme por dentro de los pantalones y en caso de una longitud excesiva de 
estos, recoger el bajo dentro de los calcetines. Utilizar calzado sin orificios.

- Posteriormente poner el equipo de protección personal (EPP) con el gorro/capuchón cerrado, mascarilla de protección, guantes internos, bata, guantes externos y gafas o pantallas.

- La anamnesis debe ser lo más concreta y concisa posible, el examen físico dirigido. Para la exploración del abdomen se deben seguir las pautas habituales.

- Al finalizar, retirar EPP por fases, idealmente ayudado. Siempre llevar a cabo lavado de manos posterior a la exposición.

- Recomiendan avisar vía telefónica a la familia y el paciente el manejo a seguir, para evitar exposiciones innecesarias.

- Respecto al consentimiento informado, se recomienda "dejar por escrito en el programa informático, tal y como se realiza de forma habitual y especificar que no se puede realizar una firma de los documentos por motivos de seguridad".

\section{Ayudas diagnósticas en COVID-19 o sospecha}

\section{Pruebas de detección rápida}

Se trata de un tipo de pruebas serológicas que se caracterizan por su alta sensibilidad y especificidad, con una notoria capacidad para detectar pacientes contagiados por el virus, pero que por sí sola no representa ser suficiente para el diagnóstico de los pacientes, razón por la cual debe apoyarse de una prueba confirmatoria, que por recomendación del Ministerio de Protección Social en la circular emitida el 25 de marzo de 2020, es la RT-PCR 5 . Justifican este hecho, dado que las pruebas de detección rápida aún están en investigación.

En caso de ser positiva la prueba de detección rápida, debe realizarse la prueba confirmatoria con RT-PCR. Si la prueba es negativa no se requiere RT-PCR, sin embargo, sigue siendo obligatorio el aislamiento por mínimo I5 días y vigilancia según la evolución. Si la exposición o el nexo epidemiológico son confirmados, la conducta diagnóstica es la realización directa de RT-PCR .

Actualmente se cuenta con dos tipos de pruebas de detección rápida:

- Prueba rápida de antígeno: detecta proteínas de la cápside viral y permite la identificación de pacientes contagiados 2 días después de haberse establecido la infección en el mejor de los casos, lo que se define como el período de ventana mínimo. Esta es la prueba de elección (ideal) para el uso masivo con fines de contención. La muestra se toma de exudado nasofaríngeo o esputo y el plazo de entrega es de Io a I5 minutos después de recogida la muestra ${ }^{6}$.

- Prueba rápida de detección de anticuerpos IgG e IgM generados por el paciente al producirse el contagio: permite identificar pacientes infectados entre el día 6 y el día 7 después de haberse producido el contagio. La muestra proviene de sangre capilar y el plazo de entrega es de aproximadamente io a I5 minutos después de haberse recogido la muestra. Entre las limitaciones de esta última prueba se encuentra que es una prueba cualitativa no cuantitativa ?

\section{Reacción en cadena de polimerasa con transcriptasa reversa (RT-PCR)}

Esta prueba identifica el gen $R d r p$ de la transcriptasa reversa y dos genes de proteínas estructurales, una nucleoproteína $(\mathrm{N})$ y una de envoltura (E). Esta prueba reporta: positivo, si se identifican los 3 genes; no concluyente, si no se encuentra el de la transcriptasa o alguna de las proteínas estructurales; y negativa, si no encontró ninguno. La prueba que se está realizando en Colombia cuenta con un valor de concordancia positiva del IOo \% (IC ${ }_{95 \%}: 92,75 \%$ - IOO\%) y de concordancia negativa de $94 \%$ ( $\mathrm{IC}_{95 \%}: 87,40 \%$ - 97,77\%) para muestras del tracto respiratorio superior ${ }^{8}$. Aunque la muestra se puede tomar de varias zonas del tracto respiratorio, idealmente debería hacerse de un aspirado traqueal o hisopado naso 
u orofaríngeo, debido a que la sensibilidad de la prueba varía por múltiples razones (hasta en 60 $\%)$, como son: el día del cuadro clínico en el que se toma, la temperatura a la que se encuentra la muestra, la ingesta de antirretrovirales, el daño de las máquinas procesadoras $\mathrm{y}$, sobre todo, el sitio de toma de la muestra 9 .

La distribución virológica en las diferentes muestras de pacientes con COVID-I9, evidencia mayor presencia del virus en el lavado broncoalveolar (93\%), seguido del esputo (72\%), hisopado nasal (63\%), biopsia por cepillado en fibrobroncoscopia (46\%), hisopado faríngeo (32\%), heces (29 \%) y sangre (I \%). Sin embargo, no es recomendable la toma de muestra del esputo inducido debido al alto riesgo de formación de aerosoles ${ }^{\text {I0 }}$.

El mayor problema que tiene esta prueba es que hasta un II, $6 \%$ a I6,6 \% de los pacientes que inicialmente tenían PCR negativa, seguían siendo considerados con alta sospecha de ser COVID-I9 positivos y, de estos, hasta el $93 \%$ se convirtieron a PCR positivas, con un promedio de 5,I \pm I. 5 días antes de positivar la prueba. Esto se debe al comportamiento del virus, que aumenta de manera progresiva el ARN y los antígenos, con pico entre el día 5 y Io, y posterior disminución, por eso, se pueden obtener falsos negativos al comienzo de la enfermedad y en la fase de recuperación. Sin embargo, la RT-PCR es la herramienta que podría detectar pacientes sin síntomas, es decir, entre el día o al 5 desde el contagio. Debido a esto, varias publicaciones concluyen que un resultado de PCR negativo no puede descartar por completo una infección por este virus y por ende, no debe ser la única prueba utilizada para tomar decisiones, y que la decisión de repetir la prueba depende de cada autoridad de salud pública ${ }^{\text {II. }}$

\section{Imágenes diagnósticas}

Estas ayudas en pacientes con sospecha o diagnóstico de COVID-I9 son de gran utilidad para su enfoque y pronóstico. Sin embargo, es imprescindible aclarar que, el diagnóstico del COVID-ı9 no es imaginológico y la confirmación con otros métodos de laboratorio, como la RT-PCR, es requerida aún con hallazgos radiológicos sugestivos. Por esto, se describe que las imágenes son un complemento a la hora de enfocar el paciente, pero no deben ser suficientes, especialmente cuando el estado del paciente es crítico. Lo primero que se debe evitar, en lo posible, es el desplazamiento de estos pacientes. Idealmente se debe disponer de una sala específica cercana y en caso de no contar con esta, se priorizará el estudio realizado con un equipo portátil.

$\mathrm{Al}$ momento se ha identificado que, si bien la radiografía de tórax es la técnica con mayor accesibilidad, tiene menor sensibilidad y especificidad que la tomografía computarizada (TC) (69 \% vs. 9I \%, respectivamente) ${ }^{\text {I2}}$. Esto debido a que la radiografía de tórax no es sensible para la detección de la opacidad en vidrio esmerilado y puede demostrar hallazgos normales en la etapa temprana de la infección ${ }^{13}$. Aunque la TC de tórax tiene mayor eficacia en la detección de cambios pulmonares por COVID-I9, está descrito que sólo el $50 \%$ de los pacientes tendrán cambios en los dos primeros días de evolución, mientras hasta el $90 \%$ presenta hallazgos entre los días 3 y 5 desde el inicio de los síntomas. Al momento, se conoce que los cambios pulmonares son inespecíficos, pudiéndose evidenciar opacidades alveolares o mixtas sin una distribución específica que nos ayude a orientar el diagnóstico. El $59 \%$ de los pacientes puede presentar consolidación pulmonar y 4I \% de los casos, imágenes en vidrio esmerilado. Adicionalmente, la enfermedad pareciera tener un predominio por las zonas periféricas pulmonares (5I \%) y compromiso bilateral (63\%) ${ }^{\mathrm{I}}$.

Por otra parte, el American College of Radiology recomienda que la TC de tórax no debe ser usada como método de tamización ni como primera línea para el diagnóstico de la enfermedad. Debe reservarse para pacientes sintomáticos, hospitalizados y con indicaciones clínicas específicas para dicha imagen. Recomiendan tener cuidado en cuanto a la recomendación de otras guías de usar la TC en la toma de decisiones y conductas, ya que una TC normal no descarta la infección por COVID-I9, así como una TC anormal no es específica para el diagnóstico de esta infección ${ }^{14}$.

Por lo anterior, y ante los cambios constantes en el enfoque del paciente quirúrgico en este es- 
cenario, se debe individualizar cada caso. En el paciente urgente o emergente, se sugiere que ninguna imagen debe retrasar el manejo oportuno. Ante la sospecha o confirmación de COVID-I9, el personal quirúrgico debe tomar las medidas pertinentes de seguridad y bajo criterio médico definir el manejo. En el contexto electivo, será a juicio médico la realización de pruebas de laboratorio e imágenes.

\section{Cambia la rutina del cirujano}

\section{Consulta externa}

Toda consulta externa no urgente debe ser cancelada o postergada, con excepción de pacientes con síntomas activos a clasificar o que requieran manejo de heridas.

- Considerar la revisión remota cuando sea posible, y directa sólo cuando sea absolutamente necesario.

- Valorar la posibilidad de transformación a consulta telefónica todas las citas posibles y posponer las citas que requieran atención presencial en los casos en los que dicha demora no ponga en peligro la vida del paciente o pueda provocar secuelas.

- Contactar telefónicamente con los pacientes para dar los resultados de análisis anatomopatológicos, pruebas de laboratorio o pruebas radiológicas y evitar visitas innecesarias de pacientes al hospital.

- Atender al paciente en un área alejada de la zona de hospitalización, utilizando medidas de protección de aislamiento de contacto en el caso de exploraciones o curación de heridas. En la medida de lo posible, los pacientes deben entrar solos en el hospital, sin acompañante ${ }^{15}$.

\section{Cirugía electiva}

En cuanto a los procedimientos quirúrgicos electivos, hay diferentes grados de prioridad y se requiere del juicio médico para discernir entre ellos. Teniendo en cuenta que se espera que el número de pacientes contagiados por COVID-I9 que requieran atención médica aumente en las próximas semanas, se deben limitar las cirugías a aquellas patologías que sean amenazantes para la vida o deriven secuelas significativas, pacientes sintomáticos que vayan a progresar o requieran manejo urgente y pacientes oncológicos con riesgo de progresión de la malignidad, balanceando la mortalidad por COVID-I9 frente a la oncológica, sobre todo en pacientes de alto riesgo, además de dar preferencia a los enfermos que no precisen ingreso en unidad de cuidados intensivos (UCI) tras cirugía ${ }^{3}$.

Previo a esto se debe tener en cuenta el estado basal del paciente y la posibilidad de contagio intrahospitalario, ya que, por ejemplo, en Italia, varios pacientes oncológicos en el postoperatorio desarrollaron SARS grave por COVID-I9, produciendo altas tasas de mortalidad ${ }^{4}$. Un estudio retrospectivo en tres centros hospitalarios en Wuhan evaluó el desenlace en 34 pacientes llevados a cirugía electiva de complejidad alta (pancreatoduodenectomía, esofagectomía, lobectomía y resección radical rectal) quienes durante el postoperatorio se contagiaron con COVID-I9, encontrando una mortalidad operatoria de 20,6\% (7/34), que alcanzó a $44 \%$ en los pacientes con enfermedad oncológica $(n=9)$. Aunque es un estudio pequeño y con diferentes sesgos, es importante tener en cuenta estos desenlaces al momento de definir qué pacientes realmente deben ser llevados a cirugía electiva en este momento ${ }^{16}$. La Society of American Gastrointestinal and Endoscopic Surgeons (SAGES) también recomienda posponer todos los procedimientos endoscópicos electivos ${ }^{17}$.

\section{Cirugía urgente}

El American College of Surgeons (ACS) propone unas pautas adicionales para la atención quirúrgica específica ${ }^{18}$. Se pretende dar recomendaciones para el cirujano general con la finalidad de brindar la mejor atención a los pacientes que se presenten con emergencias quirúrgicas durante la pandemia actual, al tiempo que se optimicen los recursos disponibles y se preserve la salud del personal asistencial. 
Para pacientes COVID-I9 o con alta sospecha, el manejo no-quirúrgico es el ideal, siempre y cuando sea posible y seguro para el paciente. $\mathrm{Si}$ el retardo en el procedimiento prolonga la estancia hospitalaria, se debe proceder con la cirugía. También se deberá considerar el manejo quirúrgico en los pacientes en quienes falle el manejo médico.

Se plantean así, las siguientes recomendaciones sobre procedimientos específicos, sin ser éstas una "camisa de fuerza" que reemplace el juicio clínico.

\section{Cirugía según la fase del hospital}

El apartado de cirugía oncológica de la AEC ${ }^{19}$ propuso una escala de 5 fases en la evolución de la pandemia para definir el manejo quirúrgico general de los pacientes. En dicha escala, sugieren qué hacer en clínicas y hospitales según el momento por el que se está pasando, medido en función del porcentaje de pacientes hospitalizados con diagnóstico de COVID-I9 y la disponibilidad de recursos en el presente y el futuro. Para cada fase se muestran a continuación las recomendaciones de modificación en el actuar quirúrgico:

Fase I. Escenario casi-normal:

- Ingresos: pacientes ingresados por COVID-I9 son menos del $5 \%$, sin existir la necesidad de urgencias definidas.

- Recursos: no impacto en los recursos del hospital.

- Acción quirúrgica: no impacto en la actividad normal.

\section{Fase II. Escenario de alerta leve:}

- Ingresos: pacientes ingresados por COVID-I9 ocupan el 5-25\% de las camas del hospital y de las camas de UCI.

- Recursos: no impacto en los recursos del hospital, pero conlleva a tener el hospital en alerta ante la pandemia y las puertas de urgencias definidas para pacientes respiratorios y resto de pacientes.

- Acción quirúrgica: actividad restringida a pacientes urgentes y oncológicos. Valorar con los pacientes oncológicos acciones similares a la fase III en caso de previsión de curva ascendente.

\section{Fase III. Escenario de alerta media:}

- Ingresos: pacientes ingresados por COVID-I9 ocupan el 25-50 \% de las camas del hospital y de las camas de UCI.

- Recursos: impacto en los recursos del hospital, con el hospital alerta ante la pandemia y las puertas de urgencias definidas para pacientes respiratorios y resto de pacientes. UCI y plantas de hospitalización reservadas para pacientes COVID-I9.

- Acción quirúrgica: actividad restringida a pacientes:

- Urgentes,

- Oncológicos que no puedan ser retrasados porque su supervivencia se compromete dentro de los 3 próximos meses,

- Oncológicos que no puedan someterse a tratamiento neoadyuvante para retrasar los procesos quirúrgicos,

- Oncológicos que no conlleven estancias largas en UCI.

Fase IV. Escenario de alerta alta:

- Ingresos: pacientes ingresados por COVID-I9 ocupan el 50-75\% de las camas del hospital y de las camas de UCI.

- Recursos: impacto clave en los recursos del hospital, profesionales y camas de UCI.

- Acción quirúrgica: actividad restringida a pacientes urgentes.

Fase V. Escenario de emergencia:

- Ingresos: pacientes ingresados por COVID-I9 ocupan más de $75 \%$ de las camas del hospital y de las camas de UCI.

- Recursos: impacto clave en los recursos del hospital, profesionales y camas de UCI. Capacidad de UCI, soporte ventilatorio y recursos de quirófano limitados, o trayectoria de COVID dentro del hospital en una fase que aumenta rápidamente. 
- Acción quirúrgica: actividad restringida a urgencias en pacientes que podrían no sobrevivir si la cirugía no se realiza en pocas horas, realizando un triage preoperatorio basado en el comité de ética.

Teniendo en cuenta el mayor riesgo de infección y el peor pronóstico en pacientes con cáncer y COVID-I9, recomiendan en zonas de alta incidencia (fases II a V) una tamización preoperatoria siempre que se defina manejo quirúrgico, mediante historia epidemiológica, identificación de síntomas típicos y RT-PCR. En pacientes oncológicos infectados, recomiendan priorizar el manejo de la infección, posponiendo el manejo quirúrgico o quimioterapéutico, excepto en los casos urgentes (perforación, sangrado, sepsis, obstrucción), en quienes se debe optar por procedimientos mínimos y con menor morbilidad.

Los casos que están pendientes de cirugía y están recibiendo quimioterapia neoadyuvante, se considera que tienen mayor riesgo de complicaciones graves secundarias por la inmunosupresión. La decisión de retrasar o suspender la quimioterapia debe evaluarse de forma individual y con un grupo multidisciplinario. Adicionalmente, en quienes sea posible, se recomienda un ciclo adicional de quimioterapia con el fin de retrasar el procedimiento quirúrgico y así poder realizar la cirugía cuando la infección por $\mathrm{CO}$ VID-I9 sea menos prevalente ${ }^{19}$.

\section{Laparoscopia versus cirugía abierta: punto controvertido}

Dentro de los avances quirúrgicos del último siglo, la cirugía laparoscópica se ha convertido en el estándar de tratamiento quirúrgico de varias patologías, al demostrar menores tiempos de estancia hospitalaria y recuperación más rápida, con menor dolor e incapacidad en el posoperatorio. Una parte esencial de esta técnica quirúrgica es la implementación del neumoperitoneo para la expansión de la cavidad abdominal, permitiendo así una mejor visualización de las estructuras intraabdominales ${ }^{20}$. Secundario a esto, se plantea la exposición del personal de quirófano a partículas en aerosoles, generando una pre- ocupación adicional para el equipo quirúrgico durante esta pandemia.

En estudios previos, se ha detectado corynebacterium, virus del papiloma y virus de inmunodeficiencia humana (VIH) en el humo quirúrgico ${ }^{21-24}$. En un estudio realizado por Kwak en 20I6, también encontraron virus de la Hepatitis B en el humo quirúrgico ${ }^{23,25}$. Basados en esto, se plantea la posibilidad de encontrar coronavirus en el humo generado en cirugía. Además, se considera que después de usar electrobisturí o dispositivos ultrasónicos durante io minutos, la concentración de partículas del humo en la cirugía laparoscópica fue significativamente mayor que en la cirugía abierta, secundario a la baja movilidad de gases en el neumoperitoneo, tendiendo a concentrarse en la cavidad abdominal ${ }^{26}$. El documento de SAGES ${ }^{17}$ aclara que actualmente, no existen datos que demuestren la presencia de aerosoles del virus COVID-ı9 liberados durante la cirugía abdominal y los datos mencionados son extrapolados de la detección de otros virus en el humo quirúrgico. Dado que los estudios anteriormente mencionados no fueron realizados en COVID-I9, no hay en el momento evidencia que sugiera que se debe contraindicar la cirugía laparoscópica. La decisión de evitar la laparoscopia debe ser individualizada y comparada con el riesgo aumentar el uso de recursos en el postoperatorio si se realiza una cirugía abierta.

Zheng y colaboradores publicaron recientemente unas recomendaciones acerca de la cirugía laparoscópica durante la pandemia por la presencia del SARS CoV-2 basadas en su experiencia en China e Italia ${ }^{27}$ :

- Los puertos de laparoscopia deben ser pequeños, con hemostasia controlada, para evitar fuga de aire y salpicadura de sangre.

- Usar dispositivos de succión para eliminar el humo y así evacuar el neumoperitoneo previo al retiro de los trocares, cierre de heridas, extracción de pieza quirúrgica o conversión a cirugía abierta.

- A esta recomendación se suma la Asociación Europea de Cirugía Endoscópica y Técnicas Intervencionistas (EAES) quie- 
nes elaboraron una lista de los dispositivos comerciales disponibles (20). Cabe resaltar que no hay evidencia de que esta práctica de rutina sea eficaz ${ }^{28-30}$, sin embargo, son explícitos en que se erra a favor del personal de salud.

- Utilizar un sistema cerrado de neumoperitoneo, es decir que éste entre solo por uno de los trocares y se evite abrir los otros para descomprimir la cavidad.

- Mantener la presión del neumoperitoneo en el nivel más bajo posible para lograr una adecuada exposición de la cavidad abdominal.

- Disminuir el tiempo de posición de Trendelenburg, para reducir las complicaciones cardiovasculares asociadas a la cirugía laparoscópica.

- Configurar el electrobisturí a la potencia más baja posible, disminuyendo la producción de humo quirúrgico.

- Evitar tiempos de disección largos con el electrobisturí. Con respecto a este punto, SAGES considera que el bisturí ultrasónico y los bipolares avanzados aumentan el riesgo de generación de aerosoles, por lo que recomiendan limitar su uso ${ }^{30,31}$.

Por su parte, la AEC establece que la vía laparoscópica se podría considerar como una barrera de protección entre el cirujano y la fuente de contagio pues direcciona el humo producido a través de los trocares, que pueden ser más fáciles de controlar que el humo producido en una cirugía abierta ${ }^{4}$. Pese a no haber evidencia científica sólida del manejo de esta enfermedad, menos aún en el ambiente quirúrgico, el personal de salud debe trabajar siempre pensando en el autocuidado, el cuidado de los trabajadores que lo acompañen y como siempre, el cuidado del paciente.

Agradecimientos: a los doctores Juan Esteban Botero, Diego Davila y Oscar G. Palacios-Rodriguez, Médicos especialistas de la Clínica CES, y a Luis G. Cadavid-Velasquez, Jefe del Programa de Cirugía General de la Universidad CES, en Medellín, Colombia, por su asesoría para el desarrollo de esta publicación.

\section{Cumplimiento de normas éticas}

Consentimiento informado: Esta publicación es una revisión de la literatura, y como tal no hay necesidad de un consentimiento informado ni de aprobación del Comité de Ética Institucional.

Declaración de conflicto de intereses: Los autores no declaran ningún conflicto de interés.

Fuentes de financiación: Autofinanciada.

\section{Referencias}

I. Saavedra-Trujillo CH, Acevedo-Medina, CA, Solórzano C, Medina Ramos DC, Vergara EC, Rodríguez Caicedo GA, Bravo Ojeda JS, et al. Consenso colombiano de atención, diagnóstico y manejo de la infección por SARS-COV-2/COVID-I9 en establecimientos de atención de la salud. Recomendaciones basadas en consenso de expertos e informadas en la evidencia. Asociación Colombiana de Infectología (ACIN) e Instituto de Evaluación de Nuevas Tecnologías de la Salud (IETS). Infectio. 2020;24(3)Suplemento Disponible en: http:// www.revistainfectio.org/index.php/infectio/article/ view/852/90I .

2. Ministerio de Salud y Protección Social. Boletín de prensa No III de 2020: Colombia entra en fase de mitigación de la COVID-I9.3I de Marzo de 2020. Disponible en: https://www.minsalud.gov.co/Paginas/Colombia-entra-en-fase-de-mitigacion-de-la-COVID-I9.aspx

3. Long Y, Hu T, Liu L, Chen R, Guo Q, Yang L, et al. Effectiveness of $\mathrm{N} 95$ respirators versus surgical masks against influenza: A systematic review and meta-analysis. J Evid Based Med; First published:I3 March 2020 https://doi.org/IO.IIII/jebm.I238I

4. Aranda-Narváez JM, Tallón-Aguilar L, Yáñez-Benítez C, Martín-Martín G, Jover-Navalón JM, Montón-Condón S, et al. Recomendaciones para manejo de pacientes con infección por COVID-I9 en el contexto de una intervención quirúrgica urgente o electiva. Asociación Española de Cirujanos. 2020. Disponible en: https:// www.aecirujanos. es/files/noticias/152/documentos/ Recomendaciones_caso_cirugia.pdf.

5. Ministerio de Salud y Protección Social. Circular externa No ol9 de 2020: Detección temprana de SARSCoV-2 / COVID-I9. 25 de Marzo de 2020. Disponible en: https:/www.minsalud.gov.co/Documents/20200325_Circular_or9.pdf

6. Abbott. COVID-I9 IgG/IgM Rapid Test Device (venous Whole Blood / Fingerstick Whole Blood / Serum / plasma) (Catalogue number: ICO-402). Disponible en: https://www.alere.com/en/home/product-details/ basepoint-covid-I9.html

7. SYNLAB. COVID-I9. Protocolos y opciones de cribado / diagnóstico. Europe's number one medical diagnos- 
tics provider. Disponible en: https://www.synlab.es/es/ Noticias/Noticias.aspx?idc=I779

8. Seegene. Summary of Allplex TM 20I9-nCoV Assay performance data. 2020. Disponible en: http://www. seegene.com/upload/product/Allplex_20I9_nCoV_performance_data.pdf

9. Loeffelholz MJ, Tang Y-W. Laboratory diagnosis of emerging human coronavirus infections - the state of the art. Emerging Microbes \& Infections. 2020;9:747-56. Doi: https://doi.org/IO.IO80/2222I75I.2020.I745095

Io. Wang W, Xu Y, Gao R, Lu R, Han K, Wu G, et al. Detection of sars-cov-2 in different types of clinical specimens. JAMA. 2020 Mar II. DOI: https://doi.org/IO.IOOI/ jama.2020.3786. [Epub ahead of print].

II. Lippi G, Simundic A-M, Plebani M. Potential preanalytical and analytical vulnerabilities in the laboratory diagnosis of coronavirus disease 2019 (COVID-19). Clin Chem Lab Med. 2020 Mar I6 [Online ahead of print]. Doi: https://doi.org/IO.I515/cclm-202O-0285

I2. Wong HYF, Lam HYS, Fong AH-T, Leung ST, Chin TWY, Lo CSY, et al. Frequency and distribution of chest radiographic findings in COVID-I9 positive patients. Radiology. 2020;20II60. Doi: https://doi.org/IO.II48/ radiol.2020201160

I3. Ng MY, Lee EY, Yang J, Yang F, Li X, Wang H, et al. Imaging profile of the COVID-I9 infection: Radiologic findings and literature review. Radiol Cardiothorac Imaging. 2020;2:e200034. Published Online: Feb I3 2020. DOI: https://doi.org/IO.II48/ryct.2020200034

I4. ACR Recommendations for the use of chest radiography and computed tomography (CT) for suspected COVID-I9 infection. Disponible en: https://www.acr. org/Advocacy-and-Economics/ACR-Position-Statements/Recommendations-for-Chest-Radiography-and-CT-for-Suspected-COVIDi9-Infection

I5. Aranda-Narváez JM, Tallón-Aguilar L, Yáñez-Benítez C, Martín-Martín G, Jover-Navalón JM, Montón-Condón $\mathrm{S}$, et al. Documentos de Posicionamiento y Recomendaciones del Grupo de Trabajo Cirugía-AEC-Covid-I9. Asociación Española de Cirujanos. Versión I.9. 2020. Disponible en: https://www.aecirujanos.es/files/noticias/I52/ documentos/dossier_v_I_9.pdf

I6. Lei S, Jiag F, Su W, Chen C, Chen J, Mei W, et al. Clinical characteristics and outcomes of patients undergoing surgeries during the incubation period of COVID-19 infection. EClinicalMedicine. $2020 \mathrm{Apr}$ 5;I0033I. Online ahead of print. Doi: https://doi.org/I0.IOI6/j.eclinm.2020.I0033I.

I7. Pryor A. SAGES Recommendations regarding surgical response to COVID-I9 crisis. 2020 Disponible en: https://www.sages.org/ recommendations-surgical- response-covid-I9/\#update

I8. American College of Surgeons. COVID-I9 Guidelines for triage of emergency general surgery patients. 2020. March 24:2020. Disponible en: https://www.facs. org/covid-I9/clinical-guidance/elective-case/emergency-surgery
19. Aranda-Narváez JM, Tallón Agui-ar L, Yáñez-Benítez C, Martín-Martín G, Jover-Navalón JM, Montón-Condón S, et al. Paciente oncológico y COVID-I9. Asociación Española de cirujanos. 2020. Disponible en: https:// www.aecirujanos.es/files/noticias/I52/documentos/ Pacientes_oncologicos_y_covidı__v2.pdf.

20. European Association for Endoscopic Surgery and other Interventional Techniques. Resources on smoke \& gas evacuation during open, laparoscopic and endoscopic procedures. Written on March 3I, 2020. Disponible en: https://eaes.eu/resources-on-smoke-gas-evacuation-during-open-laparoscopic-and-endoscopic-procedures/ .

2I. Levy, B. and Mobasheri, M. Principles of safe laparoscopic surgery. 20I7. Surgery (Oxford), 35(4), pp.216-219.

22. Alp E, Bijl D, Bleichrodt RP, Hansson B, Voss A. Surgical smoke and infection control. J Hosp Infect. 2006;62:I-5.

23. Kwak HD, Kim SH, Seo YS, Song KJ. Detecting hepatitis $B$ virus in surgical smoke emitted during laparoscopic surgery. Occup Environ Med. 2016;73:857-63. Doi: https://doi.org/IO.II36/oemed20I6-I03724.

24. Capizzi PJ, Clay RP, Battey MJ. Microbiologic activity in laser resurfacing plume and debris. Lasers in Surgery \& Medicine. $1998 ; 23: 172-4$.

25. Hensman C. Chemical composition of smoke produced by high-frequency electrosurgery in a closed gaseous environment. Surgical Endoscopy. 1998;I2:IOI7-I9.

26. Li CI, Pai JY, Chen CH. Characterization of smoke generated during the use of surgical knife in laparotomy surgeries. J Air Waste Manag Assoc. 2020;70;324-32. Doi: https://doi.org/Io.Io8o/I0962247.2020.1717675

27. Zheng M, Boni L, Fingerhut A. Minimally invasive surgery and the novel coronavirus outbreak: Lessons learned in China and Italy. Annals of Surgery. 2020 Mar 26 [Online ahead of print]. Doi: https://doi.org/IO.I097/ SLA.0ooooooooooo3924

28. Lore MB, Heimbuch BK, Brown TL, Wander JD, Hinrichs SH. Effectiveness of three decontamination treatments against influenza virus applied to filtering facepiece respirators. Ann Occup Hyg. 2012;56:92-IOI. Doi: https://doi-org/IO.I093/annhyg/mero54.

29. In SM, Park DY, Sohn IK, Kim CH, Lim HL, Hong SA, et al. Experimental study of the potential hazards of surgical smoke from powered instruments. Br J Surg. 20I5;IO2:I58I-86.

30. Wisniewski PM, Warhol MJ, Rando RF, Sedlacek TV, Kemp JE, Fisher JC. Studies on the transmission of viral disease via the $\mathrm{CO}_{2}$ laser plume and ejecta. J Reprod Med. 1990;35:III7-23.

3I. Brat G, Hersey SP, Chhabra K, Gupta A, Scott J. Protecting Surgical Teams during the COVID-I9 Outbreak: a Narrative Review and Clinical Considerations. Annals of surgery, Boston. 2020 Disponible en: https://journals. lww.com/annalsofsurgery/Documents/COVID\%20 Surgery_VF.pdf 